\title{
Non-Trivial Self-Similar Extinction Solutions for a 3D Hele-Shaw Suction Problem
}

\author{
G. Prokert and E. Vondenhoff
}

\begin{abstract}
We show the existence of noncircular, self-similar solutions to the threedimensional Hele-Shaw suction problem with surface tension regularisation up to complete extinction. In an appropriate scaling, these solutions are found as bifurcation solutions to a nonlocal elliptic equation of order three. The bifurcation parameter is the ratio of the suction speed and the surface tension coefficient.
\end{abstract}

Keywords. Hele-Shaw flow, bifurcation solution

Mathematics Subject Classification (2000). 35R35, 76D27, 47J15

\section{Introduction}

The problem of Hele-Shaw flow with suction in one point and surface tension regularisation consists in finding a family of moving domains $t \mapsto \Omega(t)$ in $\mathbb{R}^{3}$ and functions $p(\cdot, t): \Omega(t) \rightarrow \mathbb{R}$ such that

$$
\begin{array}{rlrl}
\Delta p & =\mu \delta & & \text { in } \Omega(t) \\
p=-\gamma \kappa & & \text { on } \Gamma(t):=\partial \Omega(t) .
\end{array}
$$

Here $\kappa$ stands for the mean curvature of the moving boundary $t \mapsto \Gamma(t)$ (taken negative if $\Omega(t)$ is convex) and $\gamma>0$ is the surface tension coefficient. The parameter $\mu>0$ denotes the suction speed and $\delta$ is the delta distribution. As usual, the evolution of the boundary is given by

$$
v_{n}=-\frac{\partial p}{\partial n}
$$

where $v_{n}$ is the normal velocity of the boundary.

G. Prokert: Department of Mathematics and Computer Science, Eindhoven University of Technology, The Netherlands; g.prokert@tue.nl

E. Vondenhoff: Department of Mathematics and Computer Science, Eindhoven University of Technology, The Netherlands; e.vondenhoff@tue.nl 
Besides liquid flow in a Hele-Shaw cell [2], the model and variations of it describe the growth of tumours [1] and porous media flow [3, 4].

Trivial solutions are given by balls around the origin whose volume decreases linearly in time, with rate $\mu$. The evolution of the domain that is initially the unit ball $\mathbb{B}^{3}$ is therefore

$$
\Omega(t)=\alpha(t) \mathbb{B}^{3},
$$

with $\alpha:\left[0, \frac{4 \pi}{3 \mu}\right) \rightarrow(0,1]$ given by $\alpha(t):=\sqrt[3]{1-\frac{3 \mu t}{4 \pi}}$. In [10] it is shown that if $\frac{\mu}{\gamma} \leq$ $\zeta_{2}:=\frac{32 \pi}{5}$ then this solution is nonlinearly stable with respect to perturbations that do not change the volume and the center of mass of the initial domain.

In this note we prove the existence of non-trivial solutions with the property

$$
\Omega(t)=\alpha(t) \Omega(0),
$$

for $\frac{\mu}{\gamma}$ near the values $\zeta_{k}:=4 \pi \frac{k^{3}+k^{2}-2 k}{k+3}, \quad k=2,3,4 \ldots$

This communication is organized as follows: in Section 2 we introduce a rescaled evolution equation (5) in a way that the trivial solutions described above are represented by trivial stationary solutions. In turn, non-trivial stationary solutions of (5) correspond to non-trivial self-similar extinction solutions of the original Hele-Shaw problem. We repeat some results from $[9,10]$ which form the framework for our considerations here. Because of the rescaling, the evolution operator depends on time. For the problem in $\mathbb{R}^{3}$, the operator scales in such a way that this time dependence occurs simply as a multiplication by a function of time. In Section 3 we will apply a well known result on "bifurcation from a simple eigenvalue". To ensure that the eigenvalue under consideration is simple, we have to restrict our basic space, thereby introducing a symmetry breaking. This approach is also used (for other free boundary problems) in $[5-7]$.

We want to point out here that the result depends crucially on the space dimension 3. This is due to the fact that only in this dimension the fundamental solution for the Laplacian has the same scaling behavior with respect to dilations as the curvature.

\section{The evolution problem}

Let $\mathbb{H}^{s}\left(\mathbb{S}^{2}\right)$ be the Sobolev space of order $s$ of functions on the unit sphere $\mathbb{S}^{2}$ in $\mathbb{R}^{3}$. We recall some constructions and propositions from [10] which form the basis for the results given in Section 3 .

We restrict ourselves to domain evolutions that can be described by a continuous function $R: \mathbb{S}^{2} \times[0, \infty) \rightarrow(-1, \infty)$ such that

$$
\Omega(t)=\Omega_{R(\cdot, t)}:=\left\{\xi \in \mathbb{R}^{3} \backslash\{0\}:|\xi|<1+R\left(\frac{\xi}{|\xi|}, t\right)\right\} \cup\{0\} .
$$


Besides $R$ we introduce $r: \mathbb{S}^{2} \times[0, \infty) \rightarrow(-1, \infty)$ by $1+r(x, t)=\frac{1+R(x, t)}{\alpha(t)}$. This definition of $r$ is equivalent to

$$
\Omega_{r(\cdot, t)}=\alpha(t)^{-1} \Omega_{R(\cdot, t)} .
$$

Let $\Omega_{R(t)}=\alpha(t) \Omega_{r(t)}$ be a solution to the Hele-Shaw problem (1)-(3). Then $r(t)$ satisfies an evolution equation of the form

$$
\frac{\partial r}{\partial t}=\frac{1}{\alpha(t)^{3}}\left(\gamma \mathcal{F}_{1}(r)-\mu \mathcal{F}_{2}(r)\right)
$$

with smooth $\mathcal{F}_{1}: \mathcal{U} \rightarrow \mathbb{H}^{s-3}\left(\mathbb{S}^{2}\right)$ and $\mathcal{F}_{2}: \mathcal{U} \rightarrow \mathbb{H}^{s-1}\left(\mathbb{S}^{2}\right)$, where $s>5$ and $\mathcal{U}$ is a certain neighbourhood of the origin in $\mathbb{H}^{s}\left(\mathbb{S}^{2}\right)$. For the precise structure of $\mathcal{F}_{1}$ and $\mathcal{F}_{2}$ and a derivation of $(5)$ we refer to [10]. (Hölder spaces are used there instead of Sobolev spaces. Formally, however, the arguments are identical.) Here it is sufficient to investigate the first Fréchet derivatives of $\mathcal{F}_{1}$ and $\mathcal{F}_{2}$.

For the equation (5), $r \equiv 0$ is a stationary solution corresponding to the shrinking ball $\Omega(t)=\alpha(t) \mathbb{B}^{3}$. On the other hand, it is clear that any stationary solution, i.e., any time independent $r$ satisfying

$$
\gamma \mathcal{F}_{1}(r)-\mu \mathcal{F}_{2}(r)=0
$$

corresponds to a solution $\Omega(t)=\alpha(t) \Omega_{r}$. This represents a self-similar solution of the original problem (1)-(3) which exists up to time $\frac{4 \pi}{3 \mu}$ when complete extinction of the domain takes place.

We introduce the Dirichlet-to-Neumann operator $\mathcal{N}: \mathbb{H}^{\sigma}\left(\mathbb{S}^{2}\right) \rightarrow \mathbb{H}^{\sigma-1}\left(\mathbb{S}^{2}\right)$, $\sigma>1$, as the operator that maps a function $h$ to $\mathcal{N} h:=\frac{\partial u}{\partial n}$, where $u$ satisfies

$$
\begin{array}{rr}
\Delta u=0 & \text { on } \mathbb{B}^{3} \\
u=h & \text { on } \mathbb{S}^{2} .
\end{array}
$$

This is a first order pseudodifferential operator on $\mathbb{S}^{2}$ whose spectrum consists of the nonnegative integers. The eigenfunctions are the spherical harmonics of corresponding degree.

The linearisations of $\mathcal{F}_{1}$ and $\mathcal{F}_{2}$ around $r \equiv 0$ are given by

$$
\begin{aligned}
& \mathcal{F}_{1}^{\prime}(0) h=\mathcal{N}\left(-\mathcal{N}^{2} h-\mathcal{N} h+2 h\right) \\
& \mathcal{F}_{2}^{\prime}(0) h=-\frac{1}{4 \pi}(\mathcal{N} h+3 h) .
\end{aligned}
$$

For this we refer to [9, Lemma 2.12] and [10, Lemma 2.5]. 


\section{Non-trivial stationary solutions via bifurcation}

Let $S_{l}$ be the space of spherical harmonics of order $l$. Define for $\sigma \geq 0$ the subspace $\mathbb{H}_{\times}^{\sigma}\left(\mathbb{S}^{2}\right)$ of $\mathbb{H}^{\sigma}\left(\mathbb{S}^{2}\right)$ consisting of those functions that are invariant with respect to rotations around the $z$-axis. It is well known that

$$
S_{l} \cap H_{\times}^{\sigma}\left(\mathbb{S}^{2}\right)=\left\langle Y_{l}^{0}\right\rangle,
$$

where $Y_{l}^{0}$ are the zonal harmonics given by $Y_{l}^{0}(\theta)=P_{l}(\cos \theta)$, where $\theta$ denotes the polar angle coordinate on $\mathbb{S}^{2}$ and $P_{l}$ are the Legendre polynomials.

The mappings $\mathcal{F}_{1}$ and $\mathcal{F}_{2}$ respect rotational symmetries. Therefore, on a suitable neighbourhood $\mathcal{U}_{\times}$of zero in $\mathbb{H}_{\times}^{s}\left(\mathbb{S}^{2}\right)$, we have a smooth mapping $\mathcal{F}_{\times, \mu}: \mathcal{U}_{\times} \rightarrow \mathbb{H}_{\times}^{s-3}\left(\mathbb{S}^{2}\right)$ given by

$$
\mathcal{F}_{\times, \mu}=\left.\left(\gamma \mathcal{F}_{1}-\mu \mathcal{F}_{2}\right)\right|_{\mathcal{U}_{\times}}
$$

We shall now state the main result of this note. We keep $s$ and $\gamma$ fixed and denote by $X_{k}$ the orthoplement of $\left\langle Y_{k}^{0}\right\rangle$ in $\mathbb{H}_{\times}^{s}\left(\mathbb{S}^{2}\right)$. Moreover, we write $\mu_{k}:=\gamma \zeta_{k}$.

Theorem 3.1. Let $k \geq 2$ be an integer. There exists a $\delta>0$ and a $\mathcal{C}^{1}$-curve $(f, m):(-\delta, \delta) \rightarrow X_{k} \times \mathbb{R}$ such that $(f(0), m(0))=\left(0, \mu_{k}\right)$ and for all $\tau \in(-\delta, \delta)$ we have

$$
\mathcal{F}_{\times, m(\tau)}\left(\tau Y_{k}^{0}+\tau f(\tau)\right)=0 .
$$

Furthermore, there is a neighbourhood of $\left(0, \mu_{k}\right)$ in $X_{k} \times \mathbb{R}$ on which any zero of $(r, \mu) \mapsto \mathcal{F}_{\times, \mu}(r)$ is either of the form $\left(\tau Y_{k}^{0}+\tau f(\tau), m(\tau)\right)$ or of the form $(0, \mu)$.

This theorem ensures the existence of non-trivial stationary solutions to (6). In particular, $Y_{k}^{0}$ gives the direction in which these solutions bifurcate from the trivial one, see Figure 1. The proof of Theorem 3.1 uses the following lemma. To simplify notation here, we define $A_{k} \in \mathcal{L}\left(\mathbb{H}_{\times}^{s}\left(\mathbb{S}^{2}\right), \mathbb{H}_{\times}^{s-3}\left(\mathbb{S}^{2}\right)\right)$ by

$$
A_{k}:=\mathcal{F}_{\times, \mu_{k}}^{\prime}(0) \text {. }
$$

Lemma 3.2. Let $k \geq 2$ be an integer. We have $\operatorname{ker} A_{k}=\left\langle Y_{k}^{0}\right\rangle$ and $R\left(A_{k}\right)$ has codimension one.

Proof. The zonal harmonics form a complete orthogonal system in $\mathbb{H}_{\times}^{s}\left(\mathbb{S}^{2}\right)$. Consequently, we get from (7), (8), and the fact that $\mathcal{N} Y_{l}^{0}=l Y_{l}^{0}$

$$
A_{k} h=\sum_{l \geq 0} g_{l}(k)\left\|Y_{l}^{0}\right\|_{0}^{-2}\left(h, Y_{l}^{0}\right)_{0} Y_{l}^{0}, \quad h \in \mathbb{H}_{\times}^{s}\left(\mathbb{S}^{2}\right),
$$

where $(\cdot, \cdot)_{0}$ denotes the usual inner product on $\mathbb{L}_{2}\left(\mathbb{S}^{2}\right),\|\cdot\|_{0}$ the corresponding norm, and $g_{l}(k)=-\gamma\left(l^{3}+l^{2}-2 l\right)+\frac{\mu_{k}}{4 \pi}(l+3)$. As $g_{l}(k)=0$ if and only if $l=k$ and $g_{l}(k) \sim-\gamma l^{3}$ for large $l$, both statements follow immediately. 

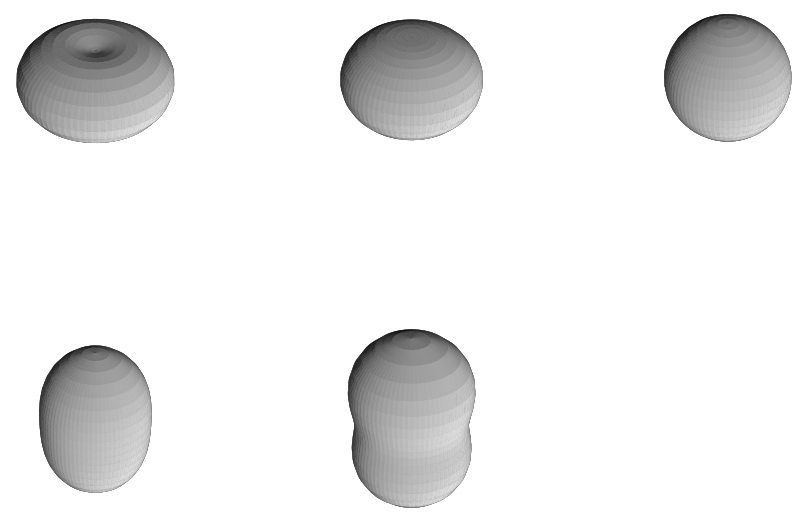

Figure 1: The domains $\Omega_{c Y_{2}^{0}}$, for several values of $c \in \mathbb{R}$. For $|c|$ small but nonzero, these domains approximate non-trivial stationary solutions of the rescaled problem, i.e., shapes of self-similarly vanishing domains.

The proof of Theorem 3.1 follows if we combine Lemma 3.2, [8, Theorem 13.5] and the fact that

$$
\left.\partial_{\mu}\left(\mathcal{F}_{\times, \mu}^{\prime}(0)\right)\right|_{\mu=\mu_{k}} Y_{k}^{0}=-\mathcal{F}_{2}^{\prime}(0) Y_{k}^{0}=\frac{k+3}{4 \pi} Y_{k}^{0} \notin R\left(A_{k}\right) .
$$

Our analysis does not provide any strict results concerning the more complicated question of stability of the solutions we found. At least for $k>2$, instability is to be expected because of the linear instability of the trivial solution for $\mu=\mu_{k}$.

Acknowledgement. The second author has been supported by NWO grant 613.000 .433$.

\section{References}

[1] Chen, X. and Friedman, A., A free boundary problem for an elliptic-hyperbolic system: an application to tumor growth. SIAM J. Math. Anal. 35 (2003)(4), $974-986$ (electronic).

[2] Elliott, C. M. and Ockendon, J. R., Weak and Variational Methods for Moving Boundary Problems. Res. Notes Math. 59. Boston: Pitman 1982.

[3] Escher, J. and Simonett, G., Maximal regularity for a free boundary problem. Nonlin. Diff. Eqs. Appl. 2 (1995)(4), $463-510$.

[4] Escher, J. and Simonett, G., Analyticity of the interface in a free boundary problem. Math. Ann. 305 (1996)(3), 439 - 459. 
[5] Fontelos, M. A. and Friedman, A., Symmetry-breaking bifurcations of charged drops. Arch. Ration. Mech. Anal. 172 (2004)(2), 267 - 294.

[6] Friedman, A. and Hu, B., Bifurcation for a free boundary problem modeling tumor growth by Stokes equation. SIAM J. Math. Anal. 39 (2007), $174-194$.

[7] Friedman, A. and Reitich, F., Symmetry-breaking bifurcation of analytic solutions to free boundary problems: an application to a model of tumor growth. Trans. Amer. Math. Soc. 353 (2001)(4), 1587 - 1634 (electronic).

[8] Smoller, J., Shock Waves and Reaction-Diffusion Equations. Grundlehren Math. Wiss. 258. New York: Springer 1983.

[9] Vondenhoff, E., Long-time behaviour of classical Hele-Shaw flows with injection near expanding balls. CASA-Report 06-19, TU Eindhoven. ftp://ftp.win.tue.nl/pub/rana/rana06-19.pdf, (2006).

[10] Vondenhoff, E., Asymptotic behaviour of injection and suction for Hele-Shaw flow in $\mathbb{R}^{3}$ with surface tension near balls. CASA-Report 06-42, TU Eindhoven. ftp://ftp.win.tue.nl/pub/rana/rana06-42.pdf, (2006).

Received June 1, 2007 